\title{
Siglec-H protects mice from lupus-like disease
}

\section{cef \\ Siglec-H is \\ known to inhibit type I}

IFN responses

5

Infection with murine cytomegalovirus (mCMV) induces severe, lupus-like autoimmune disease in mice deficient for the cell surface receptor sialic acid-binding immunoglobulin-type lectin $\mathrm{H}$ (Siglec-H), new data reveal. "We think that mCMV stimulates pattern recognition receptors on pDCs and triggers a type I IFN response, which needs to be controlled by Siglec-H," states Lars Nitschke, one of the corresponding authors on this collaborative study. "Siglec-H does not have a clear orthologue in the human, but related Siglec proteins exist that could take over this function," he notes.

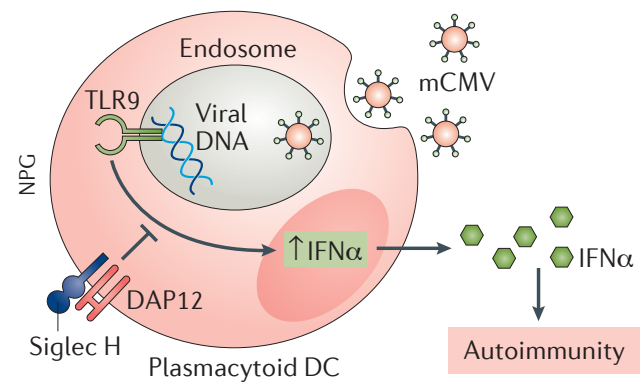

Type I IFNs have an important role in antiviral immunity and their levels are elevated in patients with systemic lupus erythematosus (SLE). Moreover, Siglec-H is known to inhibit type I IFN responses. "Siglec-H is expressed on plasmacytoid dendritic cells and on macrophage subpopulations," Nitschke explains, "so, when we observed higher type I IFN responses of Siglec-H-deficient mice after mCMV infection, we developed the hypothesis that the virus infection could lead to induction of an autoimmune disease triggered by the higher type I IFN levels."

In fact, the researchers found that elevated levels of antinuclear antibodies were present 8 weeks after mCMV infection and persisted at 26 weeks after infection in Siglec-H knockout mice, but not to the same extent in wild-type mice. Severe glomerular, tubulointerstitial and vascular pathology resembling lupus nephritis was seen in the kidneys of Siglec-H knockout mice, but not wild-type mice, at 26 weeks after infection. By contrast, doubleknockout mice lacking both Siglec- $\mathrm{H}$ and interferon- $\alpha / \beta$ receptor (the major cellular receptor for type I IFNs) developed no signs of autoimmune disease. None of the mouse models showed any evidence of mCMV reactivation after control of the initial mCMV infection.

The researchers also found that, at 26 weeks after mCMV infection, mRNA levels for the typical type I IFN-inducible genes IRF7 and CXCL10 were 10 -fold and 20 -fold higher (respectively) in splenic cells from Siglec-H knockout mice than in cells from wild-type mice, suggesting that the lack of Siglec-H leads to the induction of a chronic inflammatory response. "This is the first report, to our knowledge, that a transient virus infection in mice can lead to a severe autoimmune disease several weeks later," says Nitschke.

Joanna Collison

ORIGINAL ARTICLE Schmitt, H. et al. Siglec-H protects from virus-triggered severe systemic autoimmunity.J. Exp. Med.http://dx.doi. org/10.1084/jem.20160189(2016) 